\title{
Early pregnancy diagnosis by semiquantitative evaluation of luteal vascularity using power Doppler ultrasonography in sheep
}

\author{
Gamze Evkuran Dal ${ }^{1}$, Sinem Ozlem Enginler ${ }^{1}$, Kerem Baykal$^{2}$, Ahmet Sabuncu $^{1}$ \\ ${ }^{1}$ Istanbul University-Cerrahpasa, Faculty of Veterinary Medicine, \\ Department of Obstetrics and Gynaecology, Istanbul, Turkey, \\ ${ }^{2}$ Institute of Graduate Education, Istanbul, Turkey
}

Received November 28, 2018

Accepted February 12, 2019

\begin{abstract}
The aim of the study was to measure the area of coloured pixels captured in power Doppler examination of corpus luteum (CL) by a computer-assisted image analysis software and to evaluate the efficiency of the semiquantitative assessment of CL in early pregnancy diagnosis in sheep. Assessment of luteal vascularity was carried out on 30 Kivircik ewes with power Doppler ultrasonography daily from Day 12 to Day 19 post breeding. The mean area of coloured pixels of CL with pregnancy was found significantly higher than the area of coloured pixels of CL undergoing luteolysis $\left(0.38 \pm 0.012 \mathrm{~mm}^{2}\right.$ and $0.31 \pm 0.017 \mathrm{~mm}^{2}$, respectively, $\left.P<0.001\right)$. Assessment of day-based examination showed a significant difference between the area of coloured pixels of CL with continuing pregnancy and luteolysis on Day $17(P<0.05)$, which was followed by Days 18 and $19(P<0.001)$. It is concluded that pregnancy diagnosis can be done accurately by semiquantitative assessment of luteal vascularization as early as 17 days post breeding in ewes. According to the authors' knowledge, this is the first report on the efficacy of semiquantitative assessment of ovine CL in early pregnancy diagnosis.
\end{abstract}

Area of coloured pixels, ultrasound examination, corpus luteum, ovine

The corpus luteum (CL), formed following ovulation, synthesizes progesterone which is an essential hormone for pregnancy. The development of CL includes various steps as cellular and biochemical changes and additionally, vascular changes (Figueira et al. 2015). Angiogenesis is the further development of vessels from preexisting vascular network, and it is a relatively limited physiological process in adults with the exception of several instances as CL formation (Tamanini and De Ambrogi 2004). This functional vascular network enables CL to perform its biological role by providing oxygen, nutrients, hormones and hormonal substrates which support luteal growth and progesterone secretion (Acosta et al. 2002; Figueira et al. 2015).

Pregnancy diagnosis is one of the most important procedures in animal management and should be accurately done as early as possible. Observation of oestrus or using teaser rams to detect animals in oestrus which are not pregnant post-breeding or had an early embryonic loss are time-consuming and laborious methods (Arashiro et al. 2018). Although blood progesterone concentration measurement provides definitive diagnosis, it is not practical under field conditions due to high cost and non-real-time results. Ultrasound examination is the most widely used technique for this purpose. However, it may take up to 25 days after breeding to accurately diagnose pregnancy in ewes. Finally, the Doppler ultrasonography has become a popular and attractive tool for early pregnancy diagnosis in ovine theriogenology, as well as in veterinary medicine (Balaro et al. 2017; Arashiro et al. 2018).

Doppler ultrasonography is a highly effective noninvasive diagnostic tool which provides information about the vascularization and blood flow characteristics of the tissues that

Address for correspondence:

Ahmet Sabuncu

Department of Obstetrics and Gynaecology

Faculty of Veterinary Medicine

Istanbul University-Cerrahpasa

Avc1lar 34320, Istanbul, Turkey

Phone: $+902124737070 / 17317$

E-mail: ahmetsabuncu1968@yahoo.com

http://actavet.vfu.cz/ 
cannot be examined by B-mode ultrasonography. The Doppler shift, which is formed as a consequence of different rates of sound waves returned from the moving red blood cells, is transferred to the monitor via spectral mode or colour codes. The map, direction and velocity of blood flow can be determined in colour Doppler ultrasound system. However, power Doppler mode is preferred for tissues with very low blood flow velocity as CL (Lüttgenau and Bollwein 2014; Meinecke-Tillmann 2017; Petridis et al. 2017; Erdogan 2018).

Reproductive examinations by Doppler ultrasonography are highly current issues in farm animal practice. Although there are several reports on the haemodynamic characteristics of pregnancy in ewes (Bragança et al. 2016; Elmetwally et al. 2016; Turna Yilmaz et al. 2017; Arashiro et al. 2018), the semiquantitative evaluation of luteal vascularity data using image analysis software has not been studied before in ovine early pregnancy diagnosis.

The aim of the present study was to measure the area of coloured pixels captured in power Doppler examination of luteal tissues by computer-assisted image analysis software and to evaluate the efficiency of semiquantitative assessment of luteal tissues in early pregnancy diagnosis in sheep.

\section{Materials and Methods}

All the performed procedures were approved by the Istanbul University-Cerrahpasa Unit Ethics Committee with permit no. 2018/20.

The study was carried out at a private sheep farm located at $41^{\circ} 0^{\prime} 49.82^{\prime \prime} \mathrm{N}, 28^{\circ} 56^{\prime} 58.78^{\prime \prime} \mathrm{E}$ during the out of season period (June-July). Thirty Kivircik ewes [age: $3.03 \pm 0.49$ years old, body weight: $51.9 \pm 1.83 \mathrm{~kg}$, body condition scoring (BCS): $3.5 / 5$ ] were used in the study. Body condition scoring was done as described by Russel et al. (1969) which is accepted as BCS $=1$ for emaciated ewes to BCS $=5$ for obese ewes at 0.5 intervals. All ewes had normal clinical and gynaecological characteristics according to history findings, general clinical examinations and gynaecological examinations before entering the study. The ewes were kept under standard management conditions and were fed haylage and alfalfa. Water and mineral salt were provided ad libitum.

Ewes were subjected to an out of season synchronization protocol of melatonin + progesterone + pregnant mare serum gonadotropin (PMSG) by the farm veterinarian for the herd management program. According to the protocol, all ewes received a melatonin releasing subcutaneous ear implant (18 mg melatonin, Regulin, Ceva, Turkey). Intravaginal sponge soaked with progesterone (60 mg medroxyprogesterone acetate, Esponjavet, Hipra, Spain) was administered 35 days after melatonin implant insertion. Intravaginal sponge was removed on the $11^{\text {th }}$ day; subsequently, PMSG injection was administered intramuscularly (600 IU PMSG, Oviser, Hipra, Spain) to all ewes in the study. Controlled breeding on Day 0 was performed $48 \mathrm{~h}$ after PMSG injection. Two rams with known reproductive performance of $40-60 \%$ sperm motility were used for breeding. Rams were also subjected to a synchronization protocol with a total of 3 ear implants containing melatonin (18 $\mathrm{mg}$ melatonin, Regulin, Ceva, Turkey) at the same time with ewes.

Assessment of luteal vascularity for early pregnancy diagnosis was carried out with Doppler ultrasonography. Transrectal ultrasonographic examination of ewes was performed daily from Day 12 to Day 19 by the same operator (Arashiro et al. 2018). Ewes were maintained in the standing position. Faecal pellets were removed manually. Twenty $\mathrm{ml}$ of ultrasound gel was introduced intrarectally before insertion of the linear probe for better visualization of the tissues (Figueira et al. 2015; Petridis et al. 2017). Examination of CL were performed on power Doppler mode of a real-time B-mode ultrasound (Esaote Pie Medical MyLab Five Vet, Esaote Pie Medical, Genoa, Italy) equipped with a $6.6 \mathrm{MHz}$ linear transducer. The examination was carried out with standardised Doppler settings [pulse repetition frequency (PRF) of $1.0 \mathrm{kHz}, 7 \mathrm{~cm}$ of depth, $20 \%$ colour gain, Wall Filter (WF): medium]. Each CL was evaluated individually including CL of double ovulations.

Images with the most intensive vascularization without flash artifacts were recorded for each luteal tissue between Days 12 and 19. Computer-assisted image analysis software (PixelFlux, Chameleon Software GmbH, Münster, Germany) was used to measure the area of coloured pixels seen on the B-mode image in Power mode. Image recording and the semiquantitative analysis were performed as described by Bollwein et al. (2002).

Pregnancy diagnosis was performed on Day 19 based on the presence of gestational sac by transrectal ultrasonographic examination (Schrick and Inskeep 1993; Karen et al. 2001). Confirmation of pregnancy diagnosis was done on Day 30 by observing the embryonic heartbeat. Examinations for pregnancy diagnosis were performed by real-time B-mode ultrasound equipped with $8 \mathrm{MHz}$ linear transducer.

Statistical analysis

The SPSS 13.0 packet analysis program was used for statistical analysis. Independent Student's $t$-test was used to determine whether there was a significant difference between the mean coloured-pixel area of luteal tissues with 
continued vascularization associated with pregnancy and decreased vascularization associated with luteolysis. Analysis of data obtained on continuing days were analysed by General Linear Models (GLM) repeated measure test. The significance level was accepted as $P<0.05$.

\section{Results}

The number of pregnant and nonpregnant ewes was detected to be 21 and 9, respectively, on Day 19, and was confirmed to be the same on Day 30. Two of the 21 ewes exhibited bilateral double ovulation. One of these two ewes had two well-vascularized CL throughout the study. However, in the other double ovulated ewe, one CL continued to be vascularized while the second CL was undergoing luteolysis during the last few days of examination period. Therefore, each luteal tissue was evaluated individually (Plate I, Fig. 1) and the assessment was done according to the number of CL with continuing pregnancy $(n=22)$ and CL with luteolysis $(\mathrm{n}=10)$.

The mean area of coloured pixels of CL with pregnancy was found significantly higher than the area of coloured pixels of CL undergoing luteolysis $\left(0.38 \pm 0.012 \mathrm{~mm}^{2}\right.$ and $0.31 \pm 0.017 \mathrm{~mm}^{2}$, respectively, $\left.P<0.001\right)$.

When every single examination day was assessed separately, a significant difference between the area of coloured pixels of CL with continuing pregnancy and luteolysis was observed firstly on Day $17(P<0.05)$, followed by Days 18 and $19(P<0.001)$ as shown in Table 1.

Table 1. Mean area of coloured pixels (mean \pm S.E., $\left.\mathrm{mm}^{2}\right)$ of corpora lutea with continuing pregnancy $(\mathrm{n}=22)$ and luteolysis $(\mathrm{n}=10)$.

\begin{tabular}{lccc}
\hline Day & $\begin{array}{c}\text { Corpora lutea with } \\
\text { continuing pregnancy }\end{array}$ & $\begin{array}{c}\text { Corpora lutea with } \\
\text { luteolysis }\end{array}$ & Significance \\
\hline 12 & $0.27 \pm 0.014$ & $0.31 \pm 0.021$ & N.S. \\
13 & $0.30 \pm 0.011$ & $0.33 \pm 0.017$ & N.S. \\
14 & $0.32 \pm 0.012$ & $0.33 \pm 0.018$ & N.S. \\
15 & $0.36 \pm 0.013$ & $0.34 \pm 0.019$ & N.S. \\
16 & $0.40 \pm 0.022$ & $0.37 \pm 0.032$ & N.S. \\
17 & $0.42 \pm 0.019$ & $0.32 \pm 0.028$ & $*$ \\
18 & $0.47 \pm 0.019$ & $0.26 \pm 0.028$ & $* * *$ \\
19 & $0.52 \pm 0.022$ & $0.22 \pm 0.033$ & $* * *$ \\
\hline
\end{tabular}

S.E. - standard error; N.S. - not significant $(P>0.05)$; $: P<0.05 ; * * *: P<0.001$.

\section{Discussion}

Various methods have been defined for pregnancy detection in ewes. However, due to economical reasons it is desirable to obtain accurate results as early as possible (Karen et al. 2001). Post-breeding oestrus detection of non-pregnant ewes by teaser rams is one of the most frequently used traditional methods. In addition to being a time-consuming method, it is useless for out-of-season breeding (Arashiro et al. 2018). Real-time B-mode ultrasound examination is a non-invasive, non-expensive, easy-to-apply method, and represents real-time results. So, it is more favourable than plasma progesterone analysis. Although it is possible to detect pregnancy as early as day 13 after breeding, the most sensitive results are obtained after day 30 in ewes (Karen et al. 2001; Jones and Reed 2017). The relationship between morphological indicators of the luteal tissue and plasma progesterone 
concentrations suggests that the evaluation of CL may be effective in early detection of pregnancy. However, real-time B-mode ultrasound devices are insufficient for these evaluations (Figueira et al. 2015). Following the introduction of Doppler ultrasonography into the reproductive studies in farm animals by the 2000s, luteal vascularization was detected to decline in accordance with the decrease in progesterone concentrations during luteolysis process in cows (Lüttgenau and Bollwein 2014), in goats (Balaro et al. 2017), and finally in ewes (Arashiro et al. 2018). Although the alterations in plasma progesterone concentrations were not a topic of the present study, pregnancy CL showed a higher vascularization area than regressed ones throughout the examination period $(P<0.001)$. This finding shows compatibility with the previous reports mentioned above, and supports the notion that Doppler ultrasound examination is an effective method for pregnancy diagnosis by determining the continuing vascularity of luteal tissue which sustains pregnancy.

A decrease in luteal vascularization marks the luteolysis process which means a failure in the fertilization or pregnancy establishment process (Figueira et al. 2015; Arashiro et al. 2018). The luteal vascularity exhibited similar pattern between Days 12-16 for all ewes in the presented study. However, an increased difference was observed thereafter. The mean area of coloured pixels of CL represented an increment from Day 12 through Day 17 in all ewes, continuing until Day 19 in pregnant ewes. In nonpregnant ewes, this increase of luteal vascularization turned into a declining pattern on Day $17(P<0.05)$, and continued to decrease on Days 18 and $19(P<0.001)$. This decline of luteal vascularization was evaluated as the luteolysis process. As determination of pregnant or nonpregnant animals serves the same purpose, luteolysis of CL distinguishes the nonpregnant animal from the pregnant. In the present study, it is confirmed that early pregnancy diagnosis can be accurately done as early as Day 17 post breeding in ewes based on the Doppler ultrasonography findings. According to the results obtained in this study, the mean area of coloured pixels of $\mathrm{CL} \geq 0.42 \mathrm{~mm}^{2}$ may point out to pregnancy, whereas values below $0.40 \mathrm{~mm}^{2}$ can be a good indicator of luteolysis on Day 17 after breeding.

Similar results were observed when a very limited number of studies about the efficacy of luteal vascularization on early pregnancy diagnosis was inspected. In a review of luteal blood flow in cows, Days 15-17 after artificial insemination were stated to be important for pregnancy diagnosis according to the semiquantitative measurements obtained from power Doppler findings (Lüttgenau and Bollwein 2014). A study investigating luteal vascularization in ewes by an operator's subjective assessment has been reported recently. The researches visualized the luteal tissue with colour Doppler ultrasonography. The proportion of the luteal area was scaled as $1(0-25 \%), 2(26-50 \%), 3(51-75 \%)$ or 4 (76-100\%). The animals with a vascularization score of $\geq 2$ were assumed as pregnant. Although it was stated that false positive results may be observed as an error which originated from difficulty in distinguishing scores, nonpregnant animals could be effectively diagnosed as early as 17 days after breeding (Arashiro et al. 2018). However, it was emphasized that subjective evaluations may be influenced by the operator's experience, and the repeatability of results may be low. Consequently, it is proposed to perform evaluations with objective pixel measurements (Balaro et al. 2017). This study is the first report a semiquantitative evaluation of luteal vascularity for pregnancy diagnosis using image analysis software in ewes. The examination of CL was performed with power Doppler ultrasound due to the low blood flow velocity in CL as mentioned before (Lütthenau and Bollwein 2014; Meinecke-Tillmann 2017; Petridis et al. 2017; Erdogan 2018).

In conclusion, pregnancy diagnosis can be done accurately by semiquantitative assessment of luteal vascularization as early as 17 days post breeding in ewes. However, further studies with Doppler ultrasonography are needed to evaluate the luteolysis process during early embryonic loss. 


\section{Conflict of Interest}

The authors declare no conflict of interest.

\section{Acknowledgements}

The authors would like to express their gratitude to Assoc. Prof. Dr. Ozge Turna and Prof. Dr. Omur Kocak for their support in software analysis and statistical evaluation, respectively.

\section{References}

Acosta TJ, Yoshizawa N, Ohtani M, Miyamoto A 2002: Local changes in blood flow within the early and midcycle corpus luteum after Prostaglandin $\mathrm{F}_{20}$ injection in the cow. Biol Reprod 66: 651-658

Arashiro EKN, Ungerfeld R, Clariget RP, Pinto PHN, Balaro, MFA, Bragança GM, Riberio LSR, da Fonseca JF, Brandao FZ 2018: Early pregnancy diagnosis in ewes by subjective assessment of luteal vasularization using colour Doppler ultrasonography. Theriogenology 106: 247-252

Balaro MFA, Santos AS, Moura LFGM, Fonseca JF, Brandao FZ 2017: Luteal dynamic and functionality assessment in dairy goats by luteal blood flow, luteal biometry, and hormonal assay. Theriogenology 95: 118-126

Bollwein H, Mayer R, Weber F, Stolla R 2002: Luteal blood flow during the estrous cycle in mares. Theriogenology 65: 2043-2051

Bragança GM, Balaro MFA, Fonseca JF, Pinto PHN, Rosa RM, Ribeiro LS, Almeida MS, Souza Fabjan JMG, Garcia AR, Brandao FZ 2016: Doppler ultrasound in the diagnosis of early pregnancy in sheep. Anim Reprod 13: 587

Elmetwally M, Rohn K, Meinecke-Tillmann S 2016: Noninvasive color Doppler sonography of uterine blood flow throughout pregnancy in sheep and goats. Theriogenology 85: 1070-1079

Erdogan G 2018: Using of Doppler ultrasonography in veterinary gynecology. Turkiye Klinikleri J Vet Sci Obstet Gynecol-Special Topics 4: 43-49

Figueira LM, Fonseca JF, Arashiro EKN, Souza-Fabjan JMG, Ribeiro ACS, Oba E, Viana JHM, Brandao FZ 2015: Colour Doppler ultrasonography as a tool to assess luteal function in Santa Ines ewes. Reprod Dom Anim 50: 643-650

Jones AK, Reed SA 2017: Benefits of ultrasound scanning during gestation in the small ruminant. Small Rum Res 149: 163-171

Karen A, Kovacs P, Beckers JF, Szenci O 2001: Pregnancy diagnosis in sheep: Review of the most practical methods. Acta Vet Brno 70: 115-126

Lüttgenau J, Bollwein H 2014: Evaluation of bovine luteal blood flow by using color Doppler ultrasonography. Reprod Biol 14: 103-109

Meinecke-Tillmann S 2017: Basics of ultrasonographic examination in sheep. Small Rumin Res 152: 10-21

Petridis IG, Barbagianni MS, Ioannidi KS, Samaras E, Fthenakis GC, Vloumidi EI 2017: Doppler ultraonographic examination in sheep. Small Rumin Res 152: 22-32

Russel AJ, Doney FJM, Gunn RG 1969: Subjective assessment of fat in live sheep. J Agric Sci 72: 45-454

Schrick FN, Inskeep EK 1993: Determination of early pregnancy in ewes utilizing transrectal ultrasonography. Theriogenology 40: 295-306

Tamanini C, De Ambrogi M 2004: Angiogenesis in developing follicle and corpus luteum. Reprod Domest Anim 39: $206-216$

Turna Yilmaz O, Gündüz MC, Evkuran Dal G, Ucmak M, Günay Ucmak Z, Karacam E, Kasikci G, Kilicarslan MR 2017: Evaluation of changes in Doppler ultrasonography indices and levels of maternal serum angiogenic factors throughout pregnancy in ewes. Theriogenology 89: 183-191 
Plate I

Dal G. E. et al.: Early pregnancy ... pp. 19-23

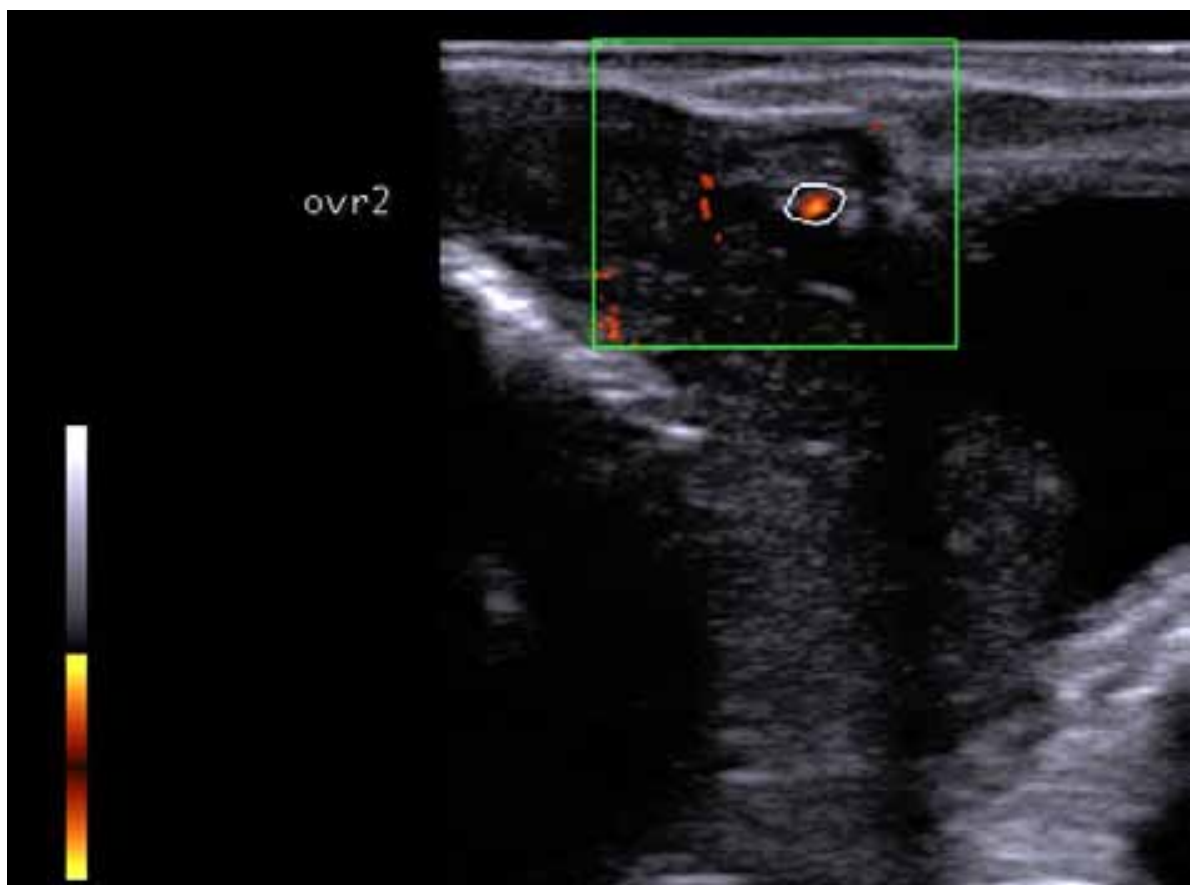

Fig. 1. Power Doppler ultrasound examination of corpus luteum in a pregnant ewe on Day 17. 